Historic, archived document

Do not assume content reflects current scientific knowledge, policies, or practices. 

TELEPHONES:

Yard and Office, Riv. $2986 \quad$ Nursery, High. 1520R1

Nursery 10 Miles South of Spokane

\section{Home Grown Trees}

\section{S. 2009 Grand Blvd., Spokane, Wn.}

\section{FRUIT DEPARTMENT}

APPLES

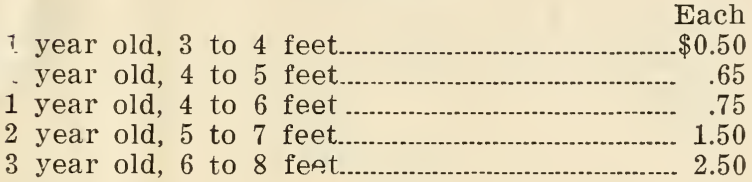

\section{Summer}

Early Harvest, Red Astrachan, Yellow Transparent, Red June

\section{Early Fall}

Duchess of Oldenburg, Jeffries, Maiden Blush, Snow, Wealthy, Gravenstein, Red Gravenstein, Mecman White, Hubenthal's Red June (a large fine red apple, the best early apple) Each

\begin{abstract}
Arkansas Black Baldwin

Delicious

Golden Russet

Grimes' Golden

Jonathan

McIntosh Red

Northern Spy

Rome Beauty
\end{abstract}

Transcendant

Hyslop's

\section{Winter}

Red Rome Beauty

Rhode Island Greening

Stayman Winesap

Spitzenburg

Talman Sweet

Wagener

Winesap

Winter Banana

Yellow Newton Pippin

\section{CRAB APPLES}

$\$ 1.00$ Each

Siberian

Whitney's No. 20

\section{PEARS}

1 year old, 4 to 6 feet

\section{Summer}

Bartlet-

Clapp's Favorite Fall

Flemish Beauty

Beurre d'Anjou

Seckle

Kieffer

Beurre Easter

Winter Bartlet

Dwarf

Bartlett

Buerre d'Anjou

\section{CHERRIES}

1 year old, 3 to 4 feet 
Bing

Lambert

\section{May Duke}

Early Richmond Montmorency

\section{Sweet}

Royal Ann

Black Tartarian

Sour

Late Duke

Olivet

English Mor

\section{PLUMS}

Each

$\$ 1.00$

1 year old, 4 to 6 feet $\$ 2.00$

2 to 3 year old

Abundance

Blue Damson

Bradshaw

Burbank

Peach Plum

Green Gage

Yellow Egg

\section{PRUNES}

Each

1 year old, 4 to 6 feet

$\$ 0.85$

2 year old, 6 to 8 feet

Italian

French Petite

Sugar

$$
\text { Silver }
$$

PEACHES

Each

2 year old

$\$ 1.00$ to $\$ 2.00$

1 year old 4 to 6 feet

Alexander

Early Crawford

Early Elberta

Elberta

\. Mounto Rose

Perfection $\$ 0.60$ to $\$ 0.85$ Indian Cling

Triumph

Nectaire

Boston

Red Beauty

\section{APRICOTS}

2 years old, selected

F-cek

2 years old, 4 to 5 feet

Moor Park

Royal

Tilton

Peach

QUINCES

2 year old

Each

Orange

Pineapple

Champion

$\$ 1.00$

\section{BUTTER NUTS}

Each

3 year old $\$ 1.75$

FIBERTS

1 year old, 2 to 4 feet

Each

$\$ 1.00$

WALNUTS

Black Walnut, 4 to 6 feet

Each

$\$ 1.00$

Black Walnut, 6 to 8 feet

2.50

Franquette, 3 to 4 feet.

GRAPES

2 year old

Each

\section{Concord}

Moore's Early

Moore's Diamond

Campbell's Early

\section{Delaware}

Watden

\section{BLACKBERRIES}

